\title{
EVALUACIÓN DE "BIOWISH REMEDIATE” EN LA BIODEGRADACIÓN DE GRASA ANIMAL EN SUELOS (VALLEDUPAR, CESAR)
}

\author{
Jessica Johana Gómez Romero ${ }^{1}$, Stefany Paola Mejía Ramírez ${ }^{2}$, Karina Paola Torres Cervera ${ }^{3}$, Pedro \\ Juan Torres Flores ${ }^{4}$.
}

\author{
${ }^{1}$ Ingeniera Ambiental y Sanitaria. Valledupar, Colombia. jessica.gomez027@gmail.com \\ 2Ingeniera Ambiental y Sanitaria. Valledupar, Colombia.stefany.mejia95@gmail.com \\ ${ }^{3}$ Ingeniera Ambiental y Sanitaria, Magíster en medio Ambiente y Desarrollo. Docente de la Universidad Popular \\ del Cesar. Valledupar, Colombia. karinaptorres@unicesar.edu.co \\ ${ }^{4}$ Licenciado en Matemáticas y física, Especialista en Educación matemáticas. Docente de la Universidad Popular \\ del Cesar. Valledupar. pedrotorres@unicesar.edu.co
}

\section{RESUMEN}

En la presente investigación se buscó evaluar la tecnología "Biowish Remediate" en la biodegradación de grasas de origen animal presentes en suelos, lo cual se realizó en tres fases metodológicas: caracterización de parámetros fisicoquímicos y microbiológicos del suelo, tasas de remoción de las grasas y determinación de efectividad de Biowish frente a un fertilizante nitrogenado. Los resultados finales para Biowish en cuanto a degradación de grasas fueron de $1350 \mathrm{mg} / \mathrm{Kg}$ de suelo (remoción del $98 \%$ ), mientras que el Fertilizante dio valores de $20750 \mathrm{mg} / \mathrm{Kg}$ (remoción 76\%). Además, al caracterizar física, química y microbiológicamente el suelo, se observó que, a pesar de que ambas alternativas se mantuvieron en igualdad de condiciones (aireación, humedad, tiempo y temperatura) para la realización de este estudio, hubo una diferencia significativa entre estas a favor de Biowish en cuanto a remoción de grasas, y en los valores finales de macronutrientes como el fósforo y el potasio.

Palabras clave: Tasas de remoción; Caracterización; Fertilizante nitrogenado, Suelo contaminado.

Recibido: 15 de abril de 2021. Aceptado: 20 de julio de 2021

Received: April 15, 2021. Accepted: July 20, 2021

DOI: https://doi.org/10.33571/rpolitec.v17n34a3

\section{EVALUATION OF "BIOWISH REMEDIATE" IN THE BIODEGRADATION OF ANIMAL FAT IN SOILS (VALLEDUPAR, CESAR)}

\begin{abstract}
In this investigation, the technology "Biowish Remediate" was evaluated in the biodegradation of animal fats present in soils, which was carried out in three methodological phases: characterization, removal rates and determination of effectiveness of Biowish versus a nitrogen fertilizer. The final results for Biowish in terms of fat degradation were $1350 \mathrm{mg} / \mathrm{kg}$ of soil, with a 98\% removal percentage, while for the Fertilizer it had values of $20750 \mathrm{mg} / \mathrm{kg}$, with a percentage of removal of $76 \%$. Also, when the soil was characterized physically, chemically and microbiologically, it was observed that, although both alternatives were maintained in the same conditions (aeration, humidity, time and temperature) for the study, there was a significant difference between these in favor of Biowish in terms of fat removal, and in the final values of macronutrients such as phosphorus and potassium.
\end{abstract}

Keywords: Removal rates; Characterization; Nitrogen fertilizer; Contaminated soil. 
Cómo citar este artículo: J.J. Gómez, S.P. Mejía, K.P. Torres, P.J. Torres. "Evaluación de "Biowish Remediate" en la biodegradación de grasa animal en suelos (Valledupar, Cesar)" Revista Politécnica, vol. 17, no. 34, pp. 39-54, 2021. DOI: https://doi.org/10.33571/rpolitec.v17n34a3

\section{INTRODUCCIÓN}

La actividad industrial ha ocasionado uno de los problemas más serios en materia de contaminación de suelos, y la ciudad de Valledupar no es la excepción, ya que es muy común encontrar zonas en las que se realizan vertimientos directos de residuos industriales al suelo, en caso específico del Municipio, residuos lácteos.

Este recurso natural, al encontrarse contaminado con este tipo de compuesto orgánico, presenta riesgo de asfixia, ya que las grasas presentes en los residuos lácteos cierran los poros del suelo e impiden el normal intercambio de gases con la atmosfera, generando malos olores [1].

El suelo para tratar, objeto de este estudio, es un suelo contaminado por grasas provenientes de una industria láctea en el predio "La Esmeralda" ubicado en la ciudad de Valledupar. Resultando importante realizar la biorremediación de este suelo, debido a que el gran contenido de materia orgánica en el efluente, así como las características propias del suelo, han reducido la acción de los microorganismos autóctonos, impidiendo la eliminación de materia orgánica presente en forma de grasa, por falta de oxígeno, esto ha llevado a que el suelo altere sus propiedades físicas y químicas y con ello su capacidad cultivable, además de la degradación del paisaje natural [2].

Una forma de evitar que estas consecuencias se sigan propagando y que se vuelvan irreversibles, es realizando una remoción que permita extraer, en su gran mayoría, las concentraciones de grasa presentes en estos suelos, presentándose como una alternativa ideal, la remediación biológica.

Este articulo busca evaluar la efectividad de la tecnología limpia Biowish Remediate, en el predio "La Esmeralda" cuyo suelo se encuentra contaminado por grasas de origen animal. Si bien el producto Biowish Remediate cuenta con una amplia experiencia en el mercado en cuanto a biorremediación, no se encontraron evidencias de que se haya probado su eficacia en cuanto a la degradación de grasas presentes en suelos, razón por la que se hace necesaria su evaluación frente a una alternativa ya comprobada en esta situación [3].

Por lo anterior se determinará la efectividad de la biodegradación en la remoción de grasas con la alternativa comercial Biowish Remediate, el cual es un producto $100 \%$ natural y no tóxico, siendo muy seguro para el uso diario en una amplia gama de productos de consumo e industriales en comparación con una alternativa convencional, que consiste en un fertilizante nitrogenado que ha sido utilizado en investigaciones anteriores obteniendo un porcentaje de remoción de grasas del $86 \%$, para que, de esta forma, se pueda seleccionar la alternativa más viable y económica para la remediación biológica de este suelo, teniendo en cuenta que las condiciones típicas de los suelos del trópico colombiano, limitan en cierta medida la producción de la mayoría de los cultivos y demandan la aplicación de grandes cantidades de fertilizantes, por tanto, se generan problemas económicos y ambientales [4], por lo que la ciudad de Valledupar se encuentra inmersa en este contexto debido a sus condiciones geográficas.

El artículo pretende recomendar la alternativa más efectiva en cuanto a tiempo, remoción y costo. De esta manera se puede lograr una degradación de estas grasas de forma efectiva generando un impacto positivo en el ambiente y dejando un suelo apto con las condiciones requeridas para que retome su productividad.

\section{MATERIALES Y MÉTODO}


Antes de aplicar los tratamientos de degradación de grasas al suelo y formar las biopilas, se procedió a homogenizar manualmente el suelo a tratar, aplanándose, con el fin de evitar desniveles en el terreno, colocando una geomembrana con el fin de evitar lixiviaciones hacia el subsuelo. Posteriormente, se tomaron muestras de este suelo contaminado a $10 \mathrm{~cm}$ de profundidad y se colocaron muestras de este $(1 \mathrm{~kg})$ en bolsas de plástico "Ziploc" para la respectiva caracterización fisicoquímica y el análisis microbiológico, tomándose de diferentes puntos y se realizó una adecuada homogenización.

Estas condiciones se analizaron antes, durante y después de la aplicación de ambas alternativas, y fueron comparadas con los parámetros evaluados e identificados de un suelo testigo.

Además, para garantizar los resultados, y para garantizar datos estadísticos confiables [5], se realizó una réplica de las muestras a todo el estudio realizado, y como resultado final se tomó un promedio entre estos. El proceso de degradación con Biowish y el fertilizante tuvo una duración de seis meses.

\section{Paso a paso del procedimiento}

Para evaluar la tecnología Biowish Remediate en la biodegradación de grasas, se realizó el proceso de degradación a través de la formación de biopilas y se trataron $105 \mathrm{Kg}$ de suelo contaminado con Biowish y $105 \mathrm{Kg}$ con el fertilizante nitrogenado, para un total de $210 \mathrm{Kg}$ de suelo contaminado tratado, y otros $210 \mathrm{Kg}$ que se usaron para realizar las réplicas. Las dimensiones de cada biopilas consistieron en medidas de $1,5 \mathrm{~m}$ $X 50 \mathrm{~cm}$, con forma rectangular.

\section{Biowish Remediate}

La aplicación del producto según su ficha técnica consistió en las siguientes actividades:

- Se mezclaron 10,5 g de Biowish ${ }^{\mathrm{TM}}$ Remediate en 10,5 $\mathrm{L}$ de agua.

- Luego se dejó por lo menos 15 a 30 minutos para la activación y posteriormente se aplicó a los 105 $\mathrm{Kg}$ de suelo contaminado.

- Posteriormente se roció el suelo afectado, para asegurar una buena dispersión a través del suelo y fue volteado manualmente (Oxígeno) como control biótico.

- Se mezclaron bien en una fuente de nutrientes microbiana incluyendo nitrógeno y fósforo, para ayudar en el proceso de degradación de la materia orgánica (3 Kg de abono orgánico).

- Durante todo el proceso de la degradación, se mantuvo el contenido de humedad en un mínimo de $40 \%$.

- Se repitió el tratamiento mensualmente hasta que se alcanzaron los niveles objetivos.

- Se evaluó el nivel de concentración de grasas, durante este proceso.

\section{Fertilizante Nitrogenado}

La aplicación del fertilizante nitrogenado, acorde con su respectiva ficha técnica, consistió en las siguientes actividades:

- En primer lugar, se mezclaron 1,63 kg de fertilizante nitrogenado con aproximadamente $40 \mathrm{~L}$ de agua.

- Posteriormente se roció esta mezcla en los $105 \mathrm{Kg}$ de suelo afectado, para asegurar una buena dispersión a través del suelo y se volteó manualmente (Oxígeno) como control biótico.

- Al mismo tiempo, se mantuvo el contenido de humedad.

- Se repitió el tratamiento mensual hasta que se alcanzaron los niveles objetivos.

- Se evaluó el nivel de concentración de grasas, durante este proceso.

Esta investigación se realizó en tres fases metodológicas con el fin de cumplir con los objetivos propuestos. La primera fue la caracterización, en donde se evaluaron los parámetros fisicoquímicos y microbiológicos que se detallan en la Tabla 1, la segunda fue la fase de tasas de remoción, en donde se calculó la cantidad de grasas degradadas mensualmente para cada tratamiento y, posteriormente, la fase de determinación de efectividad, en la que se recomendó la alternativa más efectiva en el proceso de degradación en cuanto a tasa de remoción, costo y tiempo (6 meses). 
Tabla 1. Parámetros analizados.

\begin{tabular}{|c|c|c|}
\hline ANÁLISIS & MÉTODO & LABORATORIO \\
\hline $\mathrm{pH}$ & $\begin{array}{l}\text { Potenciométrico } \quad \text { (1 suelo: } 1 \\
\text { agua) }\end{array}$ & LNS - IGAC \\
\hline Temperatura & Termómetro & En campo \\
\hline Textura & Hidrómetro de Bouyoucos & LNS - IGAC \\
\hline
\end{tabular}

\begin{tabular}{lll}
\hline Potasio & Emisión atómica - IGAC
\end{tabular}

\begin{tabular}{lll}
\hline Nitrógeno total & $\begin{array}{l}\text { Kjeldahl y } \\
\text { potenciométrica }\end{array}$ & titulación LNS - IGAC
\end{tabular}

\begin{tabular}{lll}
\hline $\begin{array}{l}\text { Capacidad de intercambio } \\
\text { catiónico (CIC) }\end{array}$ & $\begin{array}{l}\text { Acetato de amonio } 1 \mathrm{~N} \mathrm{pH} \\
\text { neutro }\end{array}$ \\
\hline Conductividad eléctrica (CE) IGAC \\
\hline Potenciométrico & LNS - IGAC
\end{tabular}

\begin{tabular}{|c|c|c|}
\hline Grasas & $\begin{array}{l}\text { Extracción Soxhlet (SM } 5520 \\
\text { D) }\end{array}$ & $\begin{array}{l}\text { Centro de Investigación de } \\
\text { Ingeniería (C.I.D.I.), } \\
\text { Facultad de ingenierías y } \\
\text { Tecnología, Universidad } \\
\text { Popular del Cesar. }\end{array}$ \\
\hline Conteo de bacterias y hongos & Vaciado de placas & $\begin{array}{l}\text { Laboratorios de } \\
\text { microbiología, } \\
\text { Departamento de ciencias, } \\
\text { Universidad Popular del } \\
\text { Cesar. }\end{array}$ \\
\hline
\end{tabular}

*LNS-IGAC *Laboratorio Nacional de Suelos -Instituto Geográfico Agustín Codazzi.

\section{RESULTADOS Y DISCUSIÓN}

Antes de aplicar el tratamiento de biorremediación al suelo y formar las biopilas, se procedió a homogenizar manualmente el suelo, y se colocaron muestras de éste en recipientes de plástico para la caracterización fisicoquímica y el análisis microbiológico.

Estas condiciones se analizaron antes, durante y después de la aplicación de ambas alternativas, y fueron comparadas con los parámetros evaluados e identificados de un suelo testigo. Además, para garantizar los resultados, se realizó una réplica a todo el estudio realizado al suelo tratado, y como resultado final se tomó un promedio entre estos. 
Esta investigación se realizó en tres fases metodológicas con el fin de cumplir con los objetivos propuestos. La primera fue la fase de caracterización, la segunda fue la fase de tasas de remoción, posteriormente la fase de determinación de efectividad de ambas alternativas.

\section{Fase 1: Caracterización}

Se hace la aclaración de que en Colombia no existe una legislación vigente con respecto a las cantidades de los parámetros analizados por lo que los resultados de estos se compararon con la tabla de interpretación de parámetros otorgada por el IGAC y, en cuanto al contenido de grasas, bacterias y hongos, se compararon valores con el obtenido en un suelo no contaminado.

- Parámetros fisicoquímicos:

\subsection{Potencial de hidrogeniones - $\mathrm{pH}$}

Durante (a los 3 meses de iniciado el proceso) y al final del proceso, se observó un aumento del pH que puede deberse al suelo calcáreo, el $\mathrm{Na}_{2} \mathrm{CO}_{3}$ y al $\mathrm{Na}^{+1}$ intercambiable en medio húmedo que producen $\mathrm{NaOH}$ [6], por lo que el pH siguió en aumento hasta 8.3, (como se puede apreciar en la Fig. 1), lo cual es un valor aceptable, teniendo en cuenta que el $\mathrm{pH}$ del suelo testigo era de 7,8 .

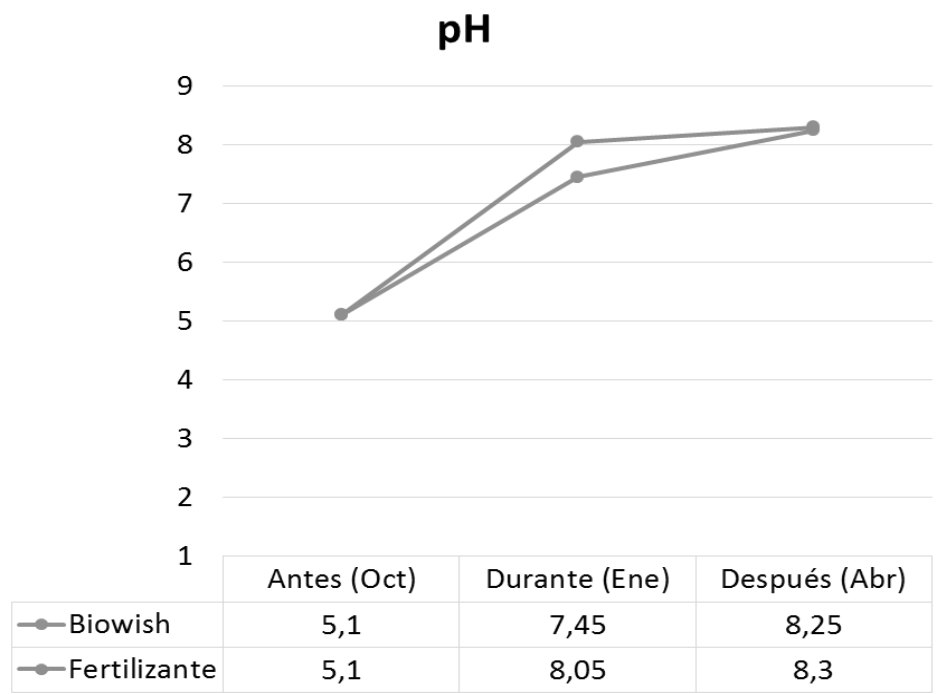

Fig. 1. Niveles de pH para Biowish y el Fertilizante nitrogenado.

Los cambios en el pH son característicos de la degradación aerobia de los contaminantes [7], y dependen de la cantidad de $\mathrm{O}_{2}$ suministrado y de los subproductos formados por la mineralización parcial o completa [8]. Cuando el oxígeno no es limitante (alta aireación), la producción de ácidos orgánicos volátiles (VOA) es baja, lo que favorece la producción de amoniaco [9] y provoca el incremento del pH, como sucedió con el trabajo desarrollado en las biopilas.

\subsection{Temperatura}

La temperatura del suelo se puede medir en su capa superficial o en profundidad; para esta investigación, la medición se realizó superficialmente. 


\section{Temperatura}

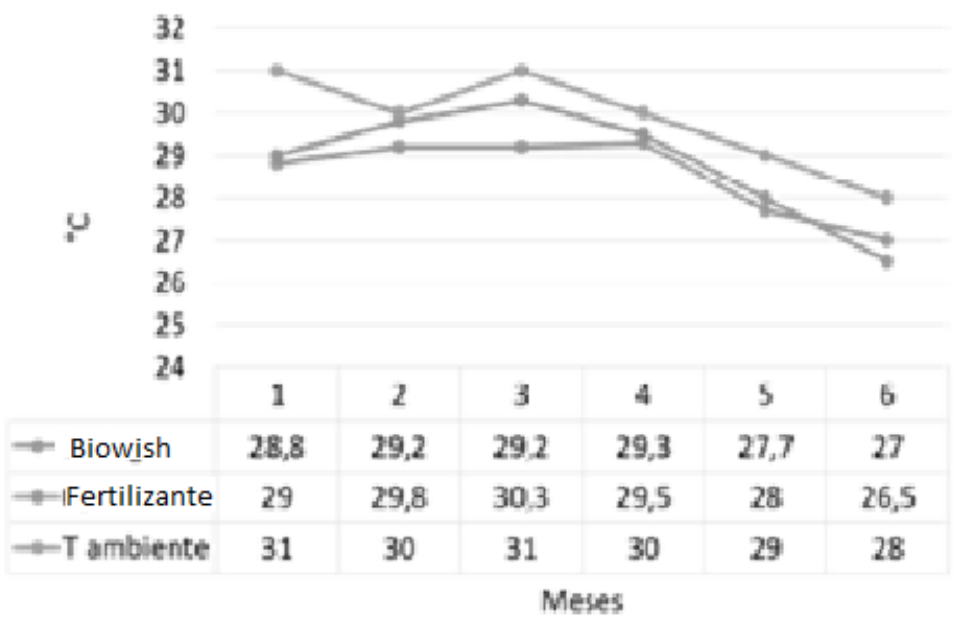

Fig. 2. Comportamiento de la temperatura en el proceso de degradación.

En la Fig. 2, se puede observar una relación muy estrecha entre temperatura ambiente y temperatura interna del suelo, durante todo el proceso, con excepción del primer mes en el que se registraron valores muy diferentes. De igual forma, es evidente, los descensos de temperatura que se registraron al final de proceso, en las cuáles tuvo mucha influencia la temperatura del lugar, la cual también disminuyó. La temperatura también se ve afectada por otros factores, como la composición del suelo, cobertura vegetal y topografía [10]. Se resalta la importancia de la temperatura interna del suelo, ya que es un factor que genera condiciones que pueden impulsar o desfavorecer el desarrollo de microorganismos.

\subsection{Textura}

La distribución de las partículas del suelo se reporta en la Fig. 3:

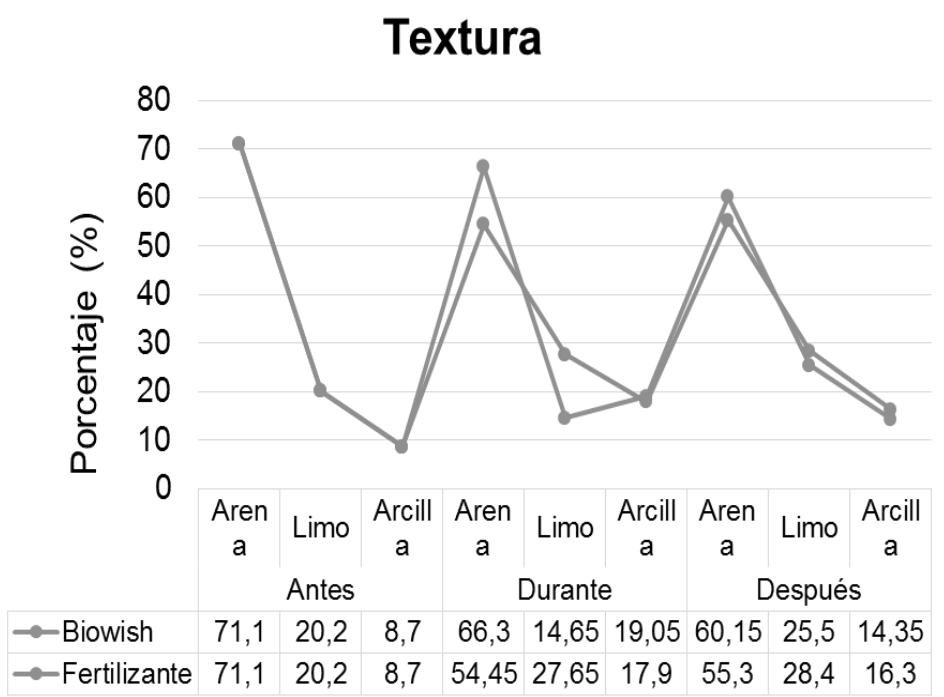

Fig. 3. Comportamiento de las partículas del suelo en la degradación.

En la Fig. 3, se puede observar que el suelo tratado con ambas alternativas presenta un comportamiento similar con respecto al porcentaje de distribución de partículas, lo que explica el que hayan mantenido la misma textura franco-arenosa durante todo el proceso de degradación, además, se debe tener en cuenta que el tiempo empleado para el estudio no es suficiente para representar un cambio relevante en las 
características de la textura. De igual forma, esta textura indica que tienen un buen drenaje y una buena aireación.

\subsection{Fósforo disponible - P}

El fósforo disponible se presenta con un valor alto en el suelo no contaminado 244,93 mg Kg-1el cual se incrementa mucho más al estar contaminado con grasas $(557 \mathrm{mg} \mathrm{Kg}-1)$ debido a la gran demanda de este parámetro para la degradación por parte de la microbiota natural del suelo. Posteriormente, este parámetro aumentó cómo se ve reflejado en la Fig. 4.

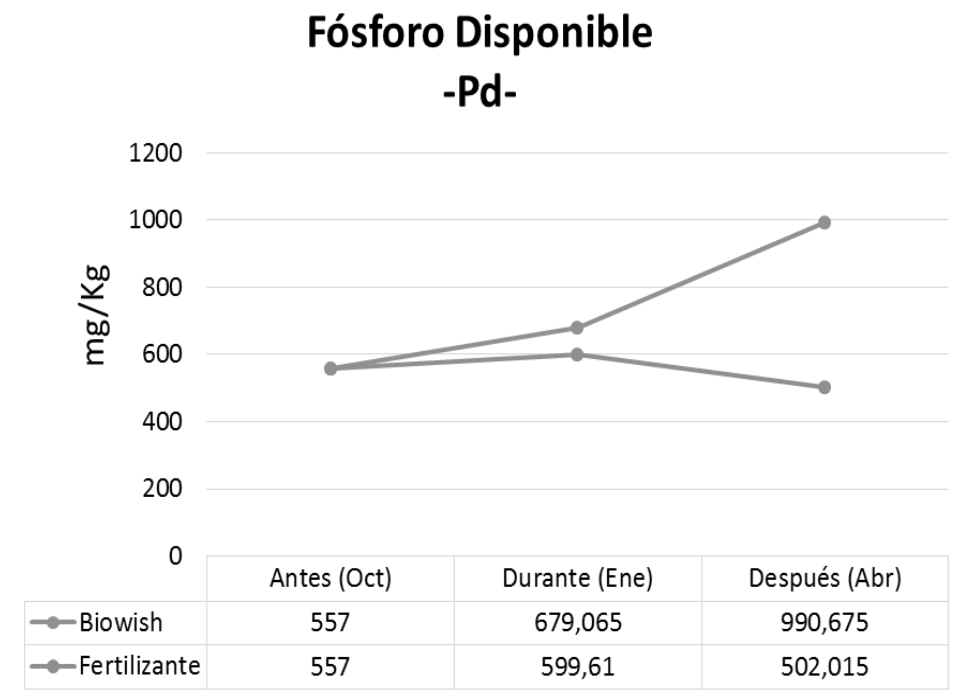

Fig. 4. Niveles de Fósforo disponible para Biowish y el Fertilizante.

Al final del proceso se observa en la Fig. 4, un aumento del fosforo disponible en el suelo tratado biológicamente que se puede deber a una liberación de fosfatos en los procesos de metabolismo microbiano, como consecuencia de la aplicación del Biowish [11], mientras que la disminución que se observa al suelo con el fertilizante, puedo deberse a la incorporación del fósforo dentro de las células microbianas [12].

\subsection{Potasio - K}

En el suelo testigo y el suelo con grasas, los análisis arrojaron un valor de 0.781 centimoles $\mathrm{cmol}(+) / \mathrm{Kg}$ y $2.641 \mathrm{cmol}(+) / \mathrm{K}$, respectivamente, siendo para ambos suelos resultados con valores altos para el potasio. Al final del proceso, el suelo tratado con el fertilizante nitrogenado, terminó el mes 6 con un valor de 1,887 cmol $(+) / \mathrm{Kg}$, obteniéndose una reducción de $2,079 \mathrm{cmol}(+) / \mathrm{Kg}$, mientras que el suelo con Biowish terminó con un valor de $3,437 \mathrm{cmol}(+) / \mathrm{Kg}$, lo cual mostró un aumento en el Potasio de este suelo, evidenciándose significativamente la diferencia entre el comportamiento del tratamiento con Biowish y el fertilizante (ver Fig. 5). 


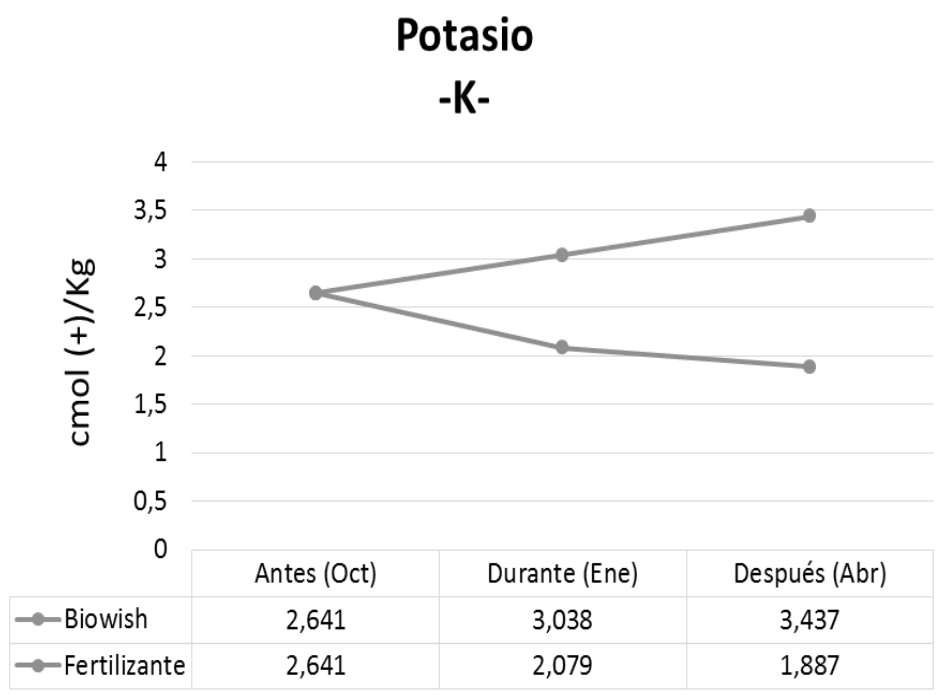

Fig. 5. Niveles de Potasio -K- para Biowish y el Fertilizante.

El potasio es movilizado por los microorganismos de acuerdo a la textura, condiciones de humedad del suelo y según sus necesidades. Además, se presenta una deficiencia de potasio en suelos con alto contenido de calcio [13], lo cual se ve reflejado en la disminución de los valores de potasio del fertilizante nitrogenado, debido a que su composición contiene Cal, siendo notable la diferencia con el tratamiento biológico.

\subsection{Nitrógeno Total - Nt}

Al finalizar la aplicación de los tratamientos, el tratamiento con Biowish arrojó un resultado de 0,7\% de nitrógeno total, mientras que, el tratamiento con el fertilizante arrojó como resultado $0,51 \%$, siendo valores normales del $0,2 \%$ al $0,7 \%$ [14], destacándose el resultado del tratamiento biológico, lo que se muestra en la Fig. 6, lo cual indica que es un suelo más rico en nitrógeno. Como es evidente, estos porcentajes de nitrógeno total superan los registrados en el suelo testigo.

Este incremento puede ser debido a un proceso de nitrificación, llevado a cabo por los microorganismos a partir del nitrógeno presente en el suelo a tratar [15].

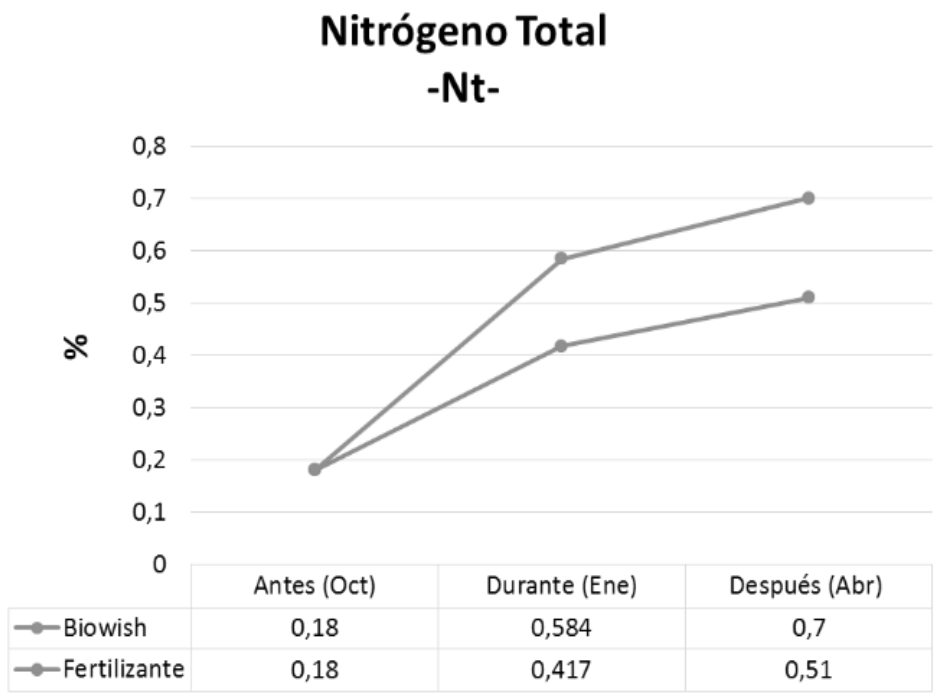

Fig. 6. Niveles de nitrógeno total para Biowish y el Fertilizante. 
Teniendo en cuenta, que el suelo tratado con Biowish, fue el que resultó con un contenido más alto de nitrógeno total al final del proceso, se puede decir que presenta las mejores condiciones para favorecer el crecimiento y desarrollo de cultivos, dependiendo de su disponibilidad, además de aportar a la fertilidad del suelo.

\subsection{Capacidad de Intercambio Catiónico - CIC}

En la Fig. 7, se muestra que no hubo variación en este parámetro al comparar los resultados del suelo testigo con el contaminado con Grasas $(9.2 \mathrm{cmol}(+) / \mathrm{Kg})$, sin embargo es un nivel bajo para este tipo de suelos.

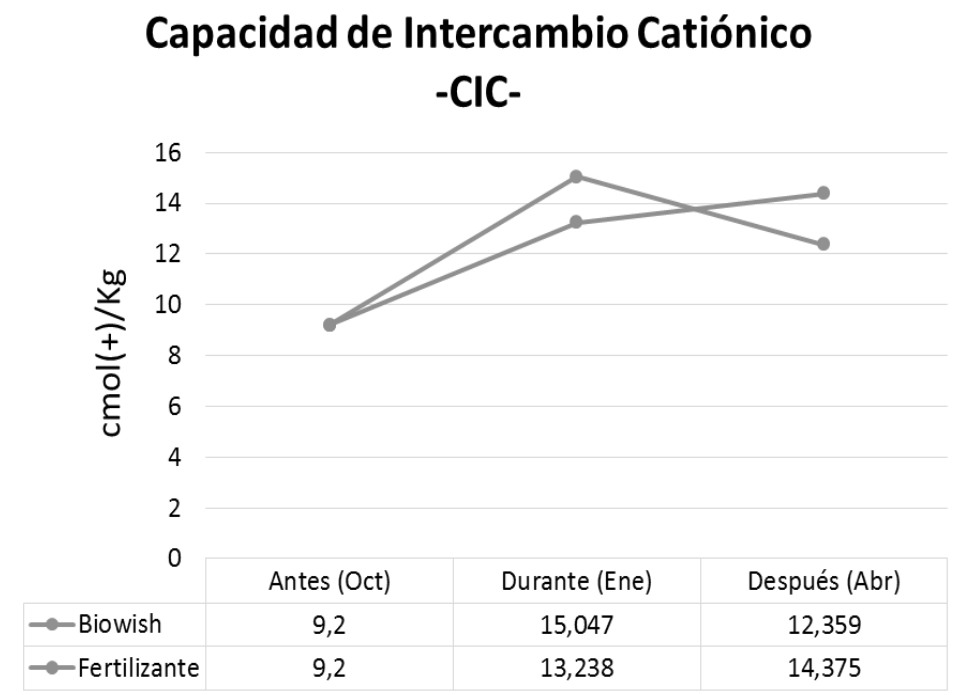

Fig. 7. Niveles de CIC para Biowish y el Fertilizante.

Ambos suelos se encuentran dentro del rango adecuado para este parámetro $(10-20 \mathrm{cmol}(+) / \mathrm{Kg})$, según la interpretación de los análisis del IGAC, por lo que, este tipo de suelo con una alta CIC pueden retener una gran cantidad de cationes y prevenir la pérdida potencial por lixiviación [16].

\subsection{Conductividad Eléctrica - CE}

Antes de iniciar el tratamiento, el suelo contenía una ligera cantidad de sales $(2,43 \mathrm{dS} / \mathrm{m})$. Por su parte, el suelo testigo registró un valor de $2,19 \mathrm{dS} / \mathrm{m}$, y a pesar de ser un valor menor que el del suelo contaminado, evidencia de todas formas, una ligera cantidad de sales también. 


\section{Conductividad eléctrica}

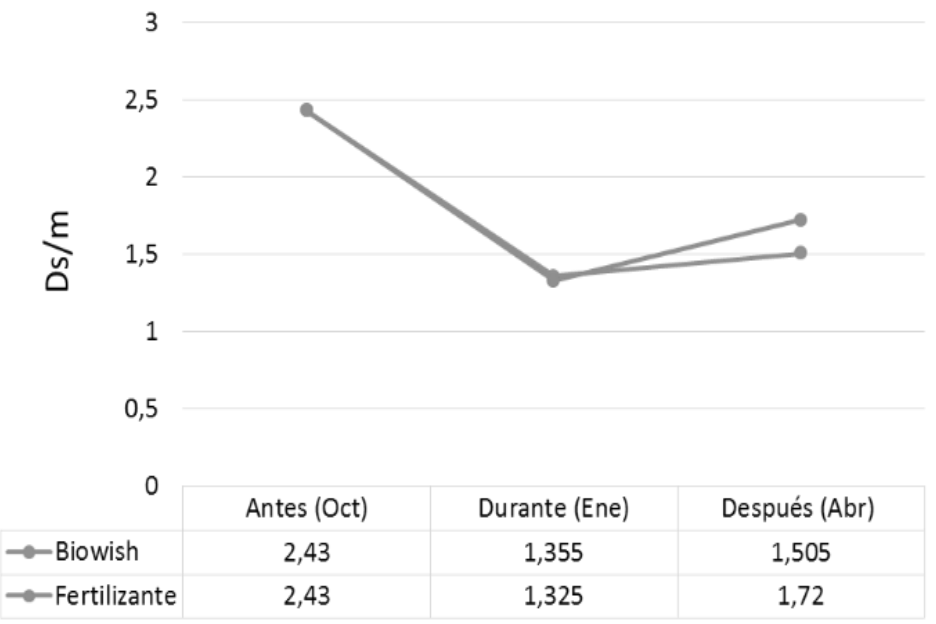

Fig. 8. Comportamiento de la CE para Biowish y el Fertilizante.

Como se puede evidenciar en la Fig. 8, los suelos tratados con ambas alternativas tuvieron un comportamiento similar durante todo el proceso de degradación. Las grasas no son polares, por lo tanto no aportan efecto sobre este parámetro; por consiguiente, la disminución de la CE durante el proceso y el leve aumento que se tuvo al finalizar se debió a la aireación constante que recibían los suelos evitando retención de sales en el mismo, así como a su textura franco-arenosa, la cual permitía un buen drenaje, por poseer partículas grandes y porosas [2].

\subsection{Grasas}

Las cinéticas muestran que la degradación de grasas fue más rápida en el primer mes para ambos tratamientos, en donde el suelo pasó de una concentración de 86238 mg Kg-1 a 45500 mg Kg-1 para Biowish y 63350 mg Kg-1, para el fertilizante_(ver Fig. 9) esto se pudo deber a la volatilización de los ácidos grasos de cadena corta y, ya en el sexto mes, se observa que los resultados finales de cantidad de grasa para el suelo tratado con Biowish fueron de $1350 \mathrm{mg} \mathrm{Kg}-1$, mientras que para el Fertilizante nitrogenado, dio valores de $20750 \mathrm{mg} \mathrm{Kg}-1$, por lo que se evidencia un mayor acercamiento a la cantidad de grasas en el suelo testigo (627 mg Kg-1) por parte de Biowish, debido a su composición biológica que aportó grandes beneficios en la degradación de dichas grasas.

\section{Contenido de grasas}

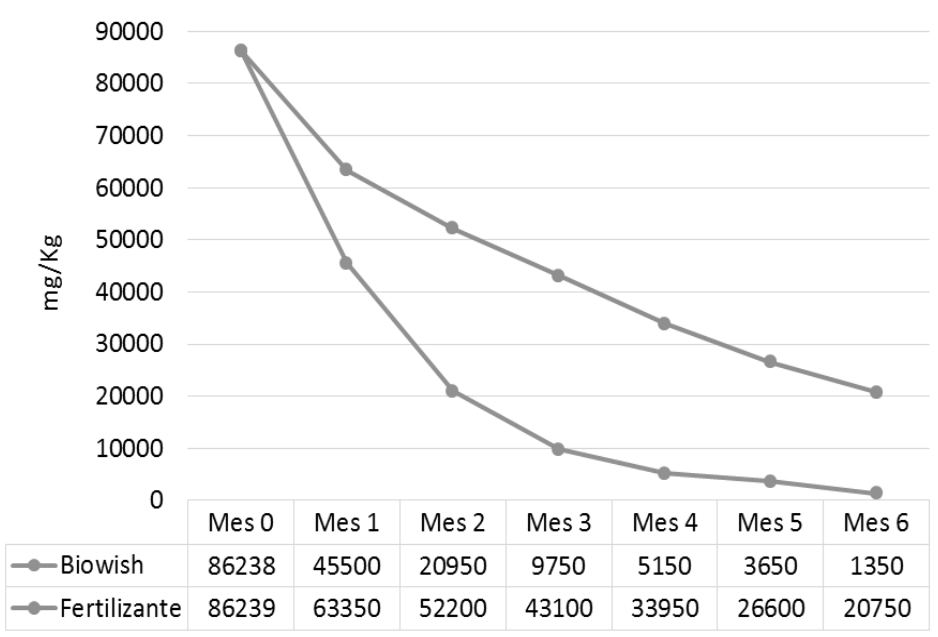


Fig. 9. Niveles de grasas para Biowish y el Fertilizante.

\section{Análisis microbiológico}

El conteo de microorganismos se llevó a cabo antes, durante y después de la aplicación de los dos tratamientos para degradar las grasas.

\subsection{Conteo de bacterias}

Antes de iniciar el proceso de degradación, se hizo conteo de bacterias a un suelo no contaminado el cual registró un resultado de 240.000 .000 U.F.C/gr de suelo.

Posteriormente, se evidencia un aumento incluso mayor en los suelos tratados, y luego un declive de la población bacteriana, lo cual probablemente sea porque la fuente de sustrato se fue agotando, sobre todo en el suelo tratado biológicamente, en el cual las grasas fueron removidas casi en su totalidad, demostrando que las bacterias fueron las principales descomponedoras de las mismas durante el proceso.

CONTEO DE BACTERIAS

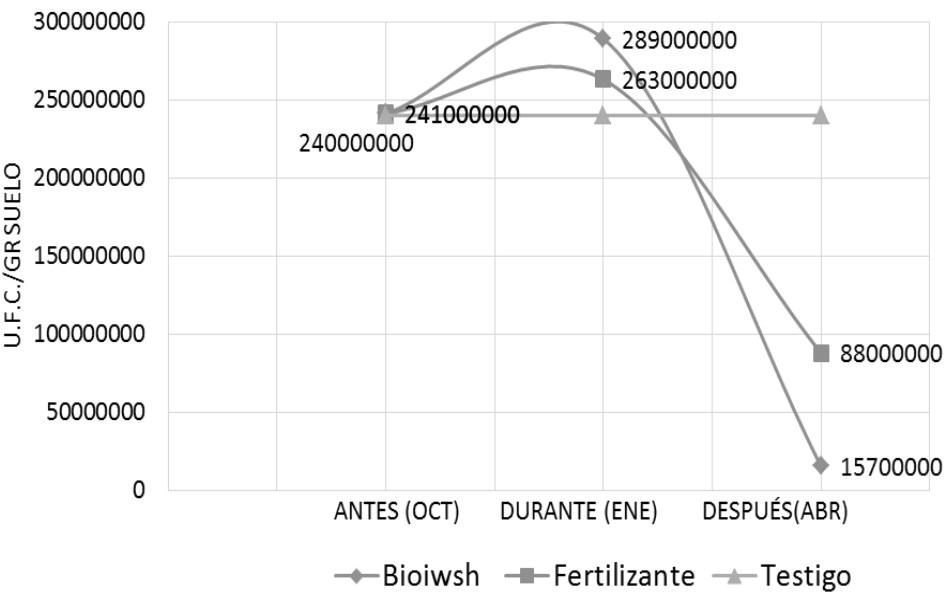

Fig. 10. Cantidad de bacterias para Biowish y el Fertilizante nitrogenado.

En la Fig. 10, se puede observar los picos más altos de crecimiento y como a partir de ese momento inicia su decrecimiento, siendo todo esto más notorio en el suelo tratado con Biowish, siendo también favorable el clima cálido, ya que las bacterias de este clima son extremadamente activas en la descomposición de materia orgánica.

\subsection{Conteo de Hongos}

Antes de iniciar el proceso de degradación, se hizo conteo de hongos a un suelo testigo o limpio el cual registró un resultado de 450.000 U.F.C/g de suelo. Se puede observar en la Fig. 11 que, al finalizar el proceso, los hongos del suelo tratados biológicamente disminuyeron un poco más- que el del suelo testigo. Sin embargo, el suelo al cual se aplicó el tratamiento químico mostró un aumento en la cantidad de hongos, más que el suelo de referencia.

Estas variaciones en la cantidad de hongos, se puede deber a las temperaturas internas del suelo que pudieron crear condiciones que favorecieron o frenaron el desarrollo de estos, ya que su crecimiento está relacionado con este factor [2]. 


\section{CONTEO DE HONGOS}

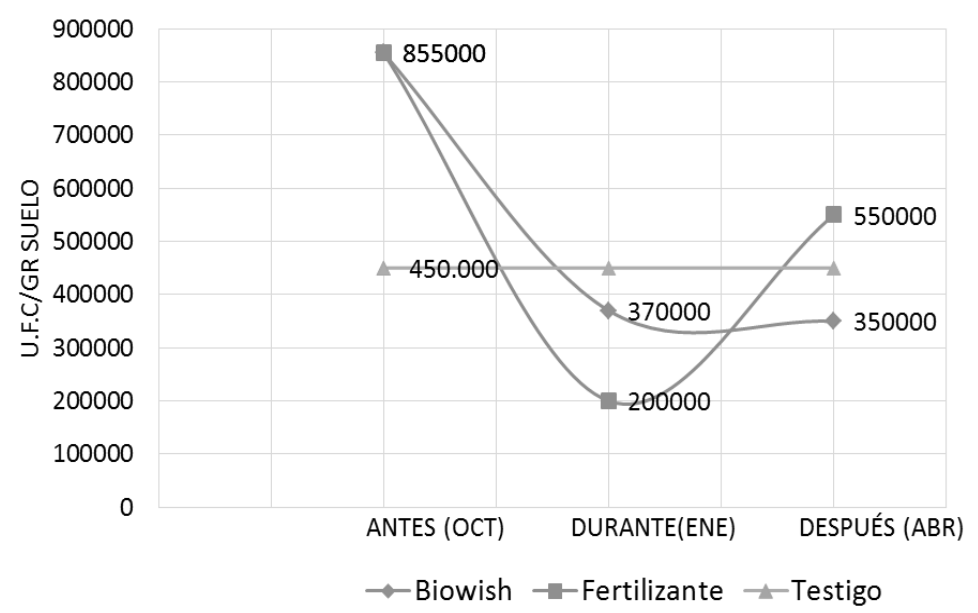

Fig. 11. Cantidad de hongos para Biowish y el Fertilizante nitrogenado.

\subsection{Fase 2: Tasa de Remoción}

De acuerdo con los resultados de las concentraciones de grasa obtenidos, se hicieron los cálculos y se obtuvo la tasa de remoción que se observa en la Tabla 2.

Tabla 2. Tasa de remoción de grasas.

\begin{tabular}{|c|c|c|c|c|c|c|}
\hline Tratamientos & $\begin{array}{l}\text { Con. } \\
\text { Ini. } \\
\text { (mg } \\
\mathrm{Kg}-1 \text { ) }\end{array}$ & $\begin{array}{l}\text { Con. } \\
\text { Fin. } \\
\text { (mg Kg- } \\
\text { 1) }\end{array}$ & $\begin{array}{l}\text { Reducció } \\
\mathrm{n} \text { en } 6 \\
\text { meses } \\
(\mathrm{mg} \mathrm{Kg}-1)\end{array}$ & $\begin{array}{l}\% \\
\text { Reducción }\end{array}$ & $\begin{array}{l}\text { Tasa de } \\
\text { remoción } \\
\text { (mg Kg-1 } \\
{ }^{*} \text { mes) }\end{array}$ & $\begin{array}{l}\text { Promedio } \\
\text { Tasa de } \\
\text { remoción } \\
\text { (mg Kg-1 } \\
\text { *mes) }\end{array}$ \\
\hline \multirow[t]{2}{*}{ Biowish 1} & 86238 & 1500 & 84738 & 98,26 & 14123,00 & \multirow{3}{*}{14148,00} \\
\hline & & & & & & \\
\hline Biowish 2 & 86238 & 1200 & 85038 & 98,61 & 14173,00 & \\
\hline Fertilizante 1 & 86238 & 21200 & 65038 & 75,42 & 10839,67 & \multirow{2}{*}{10914,67} \\
\hline Fertilizante 2 & 86238 & 20300 & 65938 & 76,46 & 10989,67 & \\
\hline
\end{tabular}

Según los datos obtenidos, Biowish degradó cada mes $14148 \mathrm{mg} \mathrm{Kg}-1$, mientras que el fertilizante nitrogenado removió 10914,67 mg Kg-1mensualmente, lo que indica una evidente ventaja de Biowish sobre el fertilizante en cuanto a remoción de grasas, en donde se considera que hubo un efecto sinérgico de la fuente de nutrientes y el producto, lo que mejoró la estructura de la matriz del suelo, aumentando su porosidad, y con ello la entrada de aire, optimizando así la remoción de grasas.

\subsection{Fase 3: Determinación de la Efectividad}

En las Fig. 12 y 13, se pueden observar la cantidad y porcentaje de remoción de grasas en función del tiempo para cada uno de los tratamientos: 


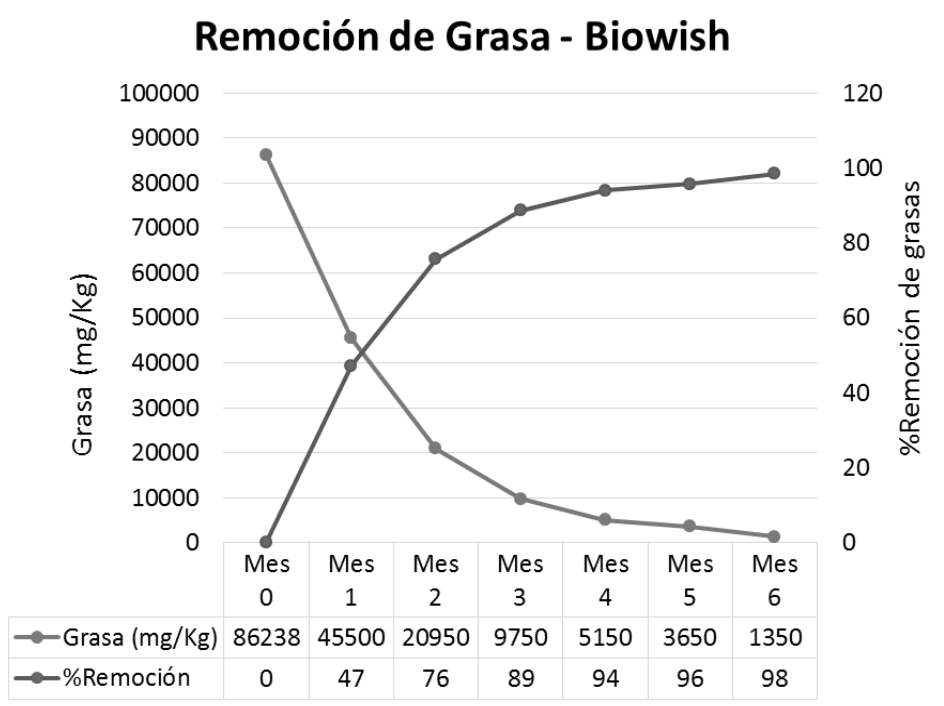

Fig. 12. Cantidad y porcentaje de remoción de grasas en función del tiempo para Biowish.

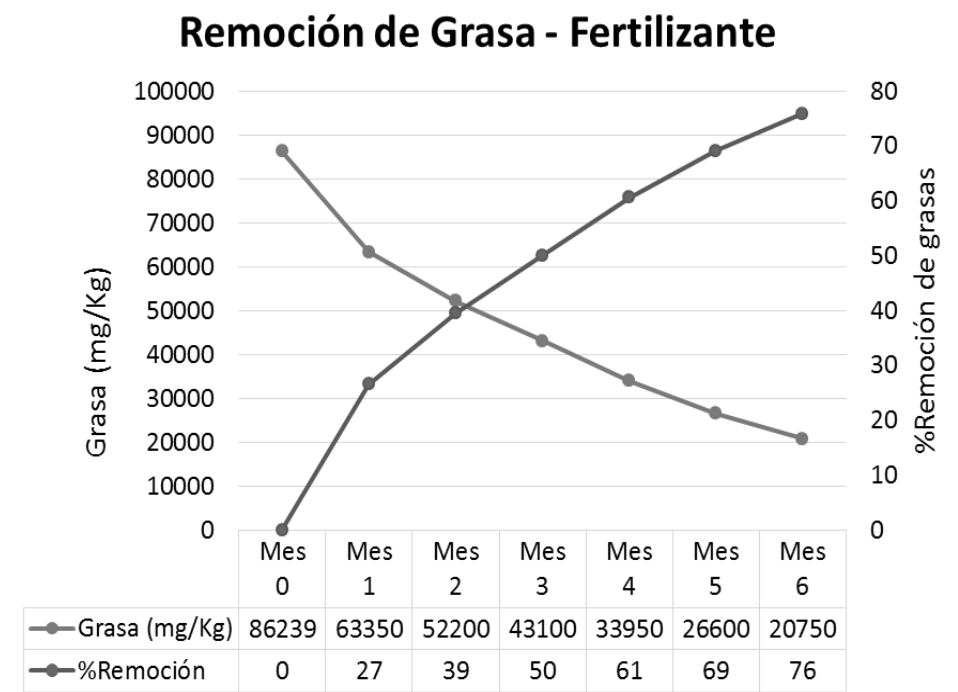

Fig. 13. Cantidad y porcentaje de remoción de grasas en función del tiempo para el Fertilizante nitrogenado.

Como se puede observar en las figuras anteriores, se evidencia la alta eficiencia que presenta Biowish en comparación con el fertilizante, ya que, en tan solo dos meses, Biowish obtiene una eficiencia del $76 \%$, la misma que alcanza el fertilizante en los 6 meses de degradación, siendo notoria la ventaja que tiene este producto biológico sobre el químico.

* Análisis de costos por producto Se calcularon los costos para cada uno de los productos, teniendo en cuenta la cantidad de suelo a tratar y la cantidad de producto necesario para este proceso según sus fichas técnicas (Tabla 3):

Tabla 3. Costo de los tratamientos y eficiencias. 


\begin{tabular}{|c|c|c|c|c|c|c|c|c|}
\hline Tratamiento & $\begin{array}{c}\text { Suelo } \\
\text { contaminado } \\
(\mathrm{kg})\end{array}$ & $\begin{array}{c}\mathrm{Kg} \\
\text { utilizados } \\
\text { en los } 6 \\
\text { meses. }\end{array}$ & $\begin{array}{c}\text { Costo } \\
\text { producto }\end{array}$ & $\begin{array}{l}\text { Costo } \\
\text { producto } \\
\text { por } \mathrm{kg} \\
\text { utilizados }\end{array}$ & $\begin{array}{l}\text { Costo } \\
\text { abono } \\
\text { orgánico }\end{array}$ & $\begin{array}{c}\text { Costo } \\
\text { abono } \\
\text { por kg } \\
\text { utilizados }\end{array}$ & $\begin{array}{c}\text { Costo total } \\
\text { del } \\
\text { tratamiento }\end{array}$ & $\begin{array}{c}\text { Eficiencia } \\
(\%)\end{array}$ \\
\hline
\end{tabular}

\begin{tabular}{lcccccccc}
\hline Biowish & 210 & 0,126 & $\$ 397.000$ & $\$ 50.000$ & $\$ 10.000$ & $\$ 7.200$ & $\$ 57.200$ & 98 \\
\hline Fertilizante & 210 & 19,56 & $\$ 80.100$ & $\$ 31.300$ & N.A. & N.A. & $\$ 31.300$ & 76 \\
\hline
\end{tabular}

Tal como se evidencia en la Tabla 3, para una misma cantidad de suelo contaminado, los $\mathrm{kg}$ utilizados por producto, es decir, por Biowish y fertilizante, fueron de $0,126 \mathrm{~kg}$ y $19,56 \mathrm{~kg}$, respectivamente. Los $\mathrm{kg}$ utilizados por Biowish son significativamente menor que los necesarios en el fertilizante.

Sin embargo, el costo de Biowish es mucho mayor que el fertilizante nitrogenado, aunque como no todos los $\mathrm{kg}$ fueron necesarios para el proceso de degradación, los costos en Biowish disminuyen notoriamente, como se puede apreciar, igualmente los del fertilizante. Teniendo en cuenta que para el tratamiento biológico fue necesaria la utilización de abono orgánico, el costo total fue de $\$ 57.200$ y para el tratamiento químico fue de $\$ 31.300$.

Haciendo una comparación, el fertilizante es \$25.900 más económico que Biowish. Sin embargo, la eficiencia del tratamiento biológico es $20 \%$ mayor que el químico, lo cual lo convierte en una opción viable en términos de eficiencia para el tratamiento de suelos contaminados con grasas.

NOTA: En el presente análisis no fueron contemplados los costos asociados a laboreo, transporte y demás, dado a que no son el objeto de esta investigación, ya que la finalidad de este análisis consiste particularmente en el precio de los productos utilizados.

Además, al realizar una comparación entre los resultados obtenidos en esta investigación y los resultados obtenidos en algunos de los antecedes consultados, como el de "Remediación de suelo contaminado con grasa del agua residual de la industria quesera"[3] y "Biorremediación para la degradación de hidrocarburos totales presentes en los sedimentos de una estación de servicio de combustible"[17], se observó que Biowish presenta la más alta eficiencia en cuanto a remoción se trata, además de brindar mejores y más óptimos resultados en parámetros como: nitrógeno, textura y fósforo.

\section{CONCLUSIONES}

Al realizar una comparación de las cinéticas de degradación de grasas, analizadas anteriormente, se puede observar que Biowish degradó en dos meses $(20950 \mathrm{mg} / \mathrm{Kg}$, con \%E=76\%), lo que el Fertilizante nitrogenado degradó en los 6 meses del tratamiento $(20750 \mathrm{mg} / \mathrm{Kg}$, con \%E=76\%), por lo que, si se quieren reducir costos en cuanto al tratamiento con Biowish Remediate, solo es cuestión de reducir el tiempo de biodegradación.

También se observa que Biowish, además de ser más efectivo que otras alternativas evaluadas, en cuanto a remoción se trata, también brinda excelentes resultados en parámetros como: nitrógeno, textura y fósforo; por lo que se recomienda el tratamiento Biológico, Biowish Remediate, para el proceso de degradación de grasas y restauración de suelos, ya que es efectivo tanto en tiempo, como en costos y en remoción. 
Técnicamente se considera que el tratamiento con Biowish fue el más efectivo, pero si bien el fertilizante no alcanzó a aproximarse al nivel de grasa del suelo limpio, este presentó una remoción del $76 \%$, lo cual puede mejorarse si se mezcla con suelo sin contaminar o se le aporta al suelo una fuente considerable de nutrientes.

\section{AGRADECIMIENTOS}

Los autores agradecen el apoyo de la Universidad Popular del Cesar a través del Centro de Investigaciones de Ingeniería y a Inversiones la Gabela S.A. por la realización de este_estudio.

\section{REFERENCIAS BIBLIOGRÁFICAS}

[1] Ardo, A. \& Beltrán J. "Ejercicios prácticos de edafología". Zaragoza: Prensas Universitarias De Zaragoza, 2015.

[2] Torres, K. y Zuluaga, T. "Biorremediación de suelos contaminados por hidrocarburos", Tesis de pregrado, Universidad Nacional de Colombia. Medellín, 2009. Recuperado de: http://www.bdigital.unal.edu.co/815/1/32242005_2009.pdf

[3] Saucedo, M. "Remediación de suelo contaminado con grasa del agua residual de la industria quesera, en la comunidad menonita de nuevo ideal, DGO”. Tesis de Maestría. Instituto Politécnico Nacional, México $\quad$ D.F., 2010. Recuperado de: http://itzamna.bnct.ipn.mx/dspace/bitstream/123456789/8212/1/REMEDSUEL.pdf

[4] Rodriguez, A., \& Sanders, I. (2016). Ciencia y tecnología Colombo-Suiza ayuda a alimentar el planeta: de la revolución verde a la revolución microbiana. Acta Biológica Colombiana, 21(1Supl), 297-303. doi:https://doi.org/10.15446/abc.v21n1Supl.50856

[5] Muskus Morales, A., Santoyo Muñoz, C., \& Plata Quintero, L. (2013). Evaluación de las técnicas de atenuación natural, bioventing, bioaumentación y bioaumentación- bioventing, para la biodegradación de diésel en un suelo arenoso, en experimentos en columna. Gestión y Ambiente, 16(2), 83-94.

de https://revistas.unal.edu.co/index.php/gestion/article/view/39565/41538

[6] Foster, A. B. Métodos aprobados de conservación de suelos. Trillas. México. 1991.

[7] Michel, F., Reddy, C. "Effect of oxygenation level on yard trimmings composting rate, odor production, and compost quality in bench scale reactor". Compost. Science \& Utilization, 6(4):614, 1998.

[8] Roldán M. Adriana, "Remoción de hidrocarburos en un sistema de biopilas con adición de texturizantes", Cinvestav, 2006.

[9] Reinhart, D., Kenneth, W.Ch. "Landfill waste pollution and control waste Management. Environ". Pollution, 15:673-674, 1995.

[10] Moreno, H. "Régimen de temperatura del suelo". [Archivo de Video].Universitat Politecnica de Valencia, 2011. Recuperado de: https://www.youtube.com/watch?v=yTXvBoQ4mhI\&t=278s [11] Si-Zhong, Y., Hui-Jun, J., Zhi, W., Rui-Xia, H., Yan-Jun, J., Xiu-Mei, L., and Shao, Y. "Bioremediation of Oil Spills in Cold Environments: A Review". Published by Elsevier Limited and Science Press. Pedosphere 19 (3): 371-381. China, 2009.

[12] Gideon, O., y Paschal, O. "A study of natural attenuation processes involved in a microcosm model of a crude oil-impacted wetland sediment in the Niger Delta". Bioresource Technology 99, 4761-4767. Nigeria, 2008. 
[13] Ríos, R. "Estudio de la Estimulación Biológica Para el Tratamiento de Residuos de Perforación Petrolera Empleando Lisímetros". Universidad Autónoma Metropolitana. Unidad Iztapalapa. Casa Abierta al Tiempo. México D.F., 2005.

[14] Costa, J. \& Ocete, C. "Efectos del nitrógeno en el suelo en la fauna edáfica". (s.f.). Recuperado de: http://www.ugr.es/ cj1/Nitrogeno\%20en\%20suelos.pdf

[15] Vallejo, V., Salgado, L., y Roldán, F. "Evaluación de la Bioestimulación en la Biodegradación de TPHs en Suelos Contaminados con Petróleo". Rev. Col. Biotec., 2 (2): 67-78, 2005.

[16] Potash \& Phosphate Institute, Foundation For Agronomic Research. "Manual de fertilidad de suelos", Canadá, 84, 1988.

[17] Nustez, D. (2012). Biorremediación para la degradación de hidrocarburos totales presentes en los sedimentos de una estación de servicio de combustible. (Tesis de Maestría. Universidad Tecnológica De Pereira, Pereira. Recuperado de: http://repositorio.utp.edu.co/dspace/bitstream/11059/2779/1/6281683N975.pdf 